\section{Inconsistent reporting about dosing, dosing regimen, and immunomodulation therapy in Pompe disease}

To the Editor: In 2001, Genetics in Medicine published an article titled: "Recombinant Human Acid a-Glucosidase Enzyme Therapy for Infantile Glycogen Storage Disease Type II: Results

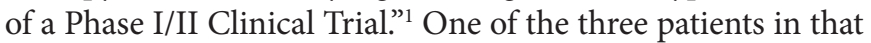
publication ("patient 1 ") was recently presented anew in a case report by Banugaria et al. ${ }^{2}$

The data presented in these two publications are mutually inconsistent. Amalfitano et al. ${ }^{1}$ published in 2001 that "patient 1 " received recombinant human acid a-glucosidase (rhGAA) in a twice-weekly dose of $5 \mathrm{mg} / \mathrm{kg}$ for 14-17 months (63-76 weeks). In contrast, Banugaria et al. ${ }^{2}$ reported that "patient 1 " received rhGAA in a twice-weekly dose of $5 \mathrm{mg} / \mathrm{kg}$ for only 5 months (22 weeks), followed by a twice-weekly dose of $10 \mathrm{mg} / \mathrm{kg}$ over the next 8 months (36 weeks), and a five-timesweekly dose of $10 \mathrm{mg} / \mathrm{kg}$ over the last 4 months (17 months in total). Furthermore, the recent case report by Banugaria et al. $^{2}$ revealed that "patient 1 " was subjected to three rounds of immunomodulation consisting of cyclophosphamide, intravenous immunoglobulin, plasmapheresis, and increased doses of rhGAA (10 mg/kg daily for 9 days in a row). According to Banugaria et al., ${ }^{2}$ the three rounds of immunomodulation occurred within the first 13 months (60 weeks) of treatment, but were not reported by Amalfitano et al. ${ }^{1}$

On another occasion, Hunley presented a case to the American College of Medical Genetics 2003 Annual Meeting. A publication followed 1 year later. ${ }^{3}$ On the basis of the age at start of treatment and the gender of the patient, the case presented by Hunley et al. ${ }^{3}$ very likely referred to "patient 2" in Amalfitano et al. ${ }^{1}$ According to Amalfitano et al., " "patient 2" received rhGAA in a twice-weekly dose of $5 \mathrm{mg} / \mathrm{kg}$ for 14-17 months, but according to Hunley et al. ${ }^{3}$ "patient 2" received rhGAA in a twice-weekly dose of $5 \mathrm{mg}$ / $\mathrm{kg}$ for only 15 weeks (3.3 months), followed by a twice-weekly dose of $10 \mathrm{mg} / \mathrm{kg}$ during the next 27 weeks (11 months), and a five-times-weekly dose of $10 \mathrm{mg} / \mathrm{kg}$ over the last 11 weeks (2.5 months) (17 months in total). Furthermore, Hunley's case report ${ }^{3}$ revealed that "patient 2" underwent experimental immune tolerance therapy beginning after 24 weeks (5.3 months) of treatment for 10 days, which consisted of frequent plasmapheresis and intravenous immunoglobulin $\mathrm{G}$ administration, with daily infusions of rhGAA $(10 \mathrm{mg} / \mathrm{kg}$ body weight) and cyclophosphamide. As with "patient 1," none of these interventions were mentioned in the article published in Genetics in Medicine. ${ }^{1}$

\begin{abstract}
DISCLOSURE
Erasmus MC and its inventors of methods to produce recombinant human acid $\alpha$-glucosidase for treatment of Pompe disease receive royalties and provide consultancy to Genzyme Corporation (Cambridge, MA) pursuant to an agreement between Genzyme Corporation and Erasmus MC.
\end{abstract}

\section{AJJ Reuser, $P h D^{1}$}

${ }^{1}$ Department of Clinical Genetics, Erasmus MC University Medical Center, Rotterdam, The Netherlands.E-mail: a.reuser@erasmusmc.nl

\section{REFERENCES}

1. Amalfitano A, Bengur AR, Morse RP, et al. Recombinant human acid $\alpha$-glucosidase enzyme therapy for infantile glycogen storage disease type II: results of a phase I/II clinical trial. Genet Med 2001;3:132-138.

2. Banugaria SG, Patel TT, Mackey J, et al. Persistence of high sustained antibodies to enzyme replacement therapy despite extensive immunomodulatory therapy in an infant with Pompe disease. Mol Genet Metab 2012;105:677-680

3. Hunley TE, Corzo D, Dudek M, et al. Nephrotic syndrome complicating $\alpha$-glucosidase replacement therapy for Pompe disease. Pediatrics 2004;114:e532-e535.

advance online publication 9 August 2012. doi:10.1038/gim.2012.80

\section{Response to Reuser}

To the Editor: We read with interest the letter by Dr Reuser titled "Inconsistent Reporting About Dosing, Dosing Regimen, and Immunomodulation Therapy in Pompe Disease." In our 2001 article, "Recombinant Human Acid $\alpha$-Glucosidase Enzyme Therapy for Infantile Glycogen Storage Disease Type II: Results of a Phase I/II Clinical Trial,"2 we had reported the initial use of recombinant human acid $\alpha$-glucosidase (rhGAA) in three infantile patients with Pompe disease.

This was a clinical study where important conclusions were drawn. At that time, we had hypothesized that the lack of sustained efficacy of rhGAA in terms of muscle strength in patients 1 and 2 after 3 to 4 months of the initiation of the therapy was likely due to onset of antibodies against rhGAA in these crossreactive immunologic material-negative patients, in contrast to the lack of an antibody response noted in the cross-reactive immunologic material-positive patient 3.

As several subsequent trials have borne out, the observations and conclusions drawn have been repeated and confirmed, both in regard to the impact that cross-reactive immunologic material-positive status with low antibody titers has on potential for significant cardiac improvement and improvement in motor strength. ${ }^{3-8}$ In fact, dosing for acid alpha glucosidase is now clinically indicated at essentially an equivalent dosing 
for all Pompe patients, albeit now being given as a total dose of $20-\mathrm{mg} / \mathrm{kg}$-every-other-week infusion, to reduce the need for frequent intravenous infusions. ${ }^{9}$ The problems of lack of sustained improvement in motor status in CRIM-negative patients with high-titer antibody have also been verified, indeed new clinical trials attempting to address this concern are ongoing. ${ }^{5,6}$

Dr Reuser is correct in noting that in our original report, we had not disclosed that in latter portions of the first clinical trial for patients 1 and 2 (who had declining AIMS scores and increasing antibody titers), that we attempted to treat these patients with increased doses of the enzyme, as well as various immunomodulatory therapies, in an effort to save these children from continuing their clinical decline. These attempts were ongoing during submission and review of our original manuscript, and were well beyond the scope of that manuscript, requiring further more detailed review and reporting as noted in subsequent publications ${ }^{10,11}$ as he has indicated. It is, however, clear that these patients had clinical improvement at the $5 \mathrm{mg} / \mathrm{kg}$ twice weekly dose before the development of high and sustained antibody titers, time points we focused upon in that manuscript. Thus the conclusions of this dosing regimen (cumulative dose of $20 \mathrm{mg} / \mathrm{kg}$ every 2 weeks) still holds true in current clinical practice.

In addition, we agree with Dr Reuser that there may be confusion regarding the sentence, "Three patients with infantile Pompe disease have been receiving twice-weekly intravenous infusions of rhGAA for 14 to 17 months," which we feel has been addressed in the corrigendum to our article (see Corrigendum in this issue), which includes the following information:

Three patients with infantile Pompe disease have been receiving at least twice-weekly intravenous infusions of rhGAA for 14-17 months. Patient 1 received $5 \mathrm{mg} / \mathrm{kg}$ twice weekly for the first 20 weeks; patient 2 received $5 \mathrm{mg} / \mathrm{kg}$ twice weekly for the first 15 weeks; and patient 3 continued to receive the 5-mg/ kg-twice-weekly dosing throughout the study. However, after these time points, and because of high antibodies, patients 1 and 2 underwent a series of attempts to induce tolerance to the rhGAA with immunomodulatory therapy and/or increased dosing of the acid alpha glucosidase, attempts that have been subsequently fully described.

\section{DISCLOSURE}

A.A. is on the speakers bureau for Genzyme Corporation; P.S.K. has received research/grant support and honoraria from Genzyme Corporation. P.S.K. is a member of the Pompe and Gaucher Disease Registry Advisory Board for Genzyme Corporation. Y.T.C. has received honoraria from Genzyme Corporation. Alglucosi- dase alfa rhGAA, in the form of Genzyme's product alglucosidase alfa, (Myozyme/Lumizyme) has been approved by the US Food and Drug Administration and the European Union as therapy for Pompe disease. Duke University and the inventors of the method of treatment and precursors of the cell lines used to generate the enzyme (rhGAA) used commercially have received royalties pursuant to the university's policy of inventions, patents, and technology transfer. This potential conflict for Duke University has been resolved through monetization.

Andrea Amalfitano, DO, $P h D^{1,2}$, Priya S. Kishnani, $M D^{3}$ and YT Chen, $M D, P h D^{4,5}$

${ }^{1}$ Department of Pediatrics, College of Osteopathic Medicine, Michigan State University, East Lansing, Michigan, USA; ${ }^{2}$ Department of Microbiology and Molecular Genetics, College of Osteopathic Medicine, Michigan State University, East Lansing, Michigan, USA; ${ }^{3}$ Division of Medical Genetics, Department of Pediatrics, Duke University Medical Center, Durham, North Carolina, USA; ${ }^{4}$ Institute of Biomedical Sciences, Academia Sinica, Taipei, Taiwan; ${ }^{5}$ Division of Medical Genetics, Department of Pediatrics, Duke University Medical Center, Durham, North Carolina, USA. Correspondence: YT Chen (chen0010@ibms.sinica.edu.tw)

\section{REFERENCES}

1. Reuser AJJ. Inconsistent reporting about dosing, dosing regimen, and immunomodulation therapy in Pompe disease. Genet Med 2012; doi:10.1038/gim.2012.80.

2. Amalfitano A, Bengur AR, Morse RP, et al. Recombinant human acid alpha-glucosidase enzyme therapy for infantile glycogen storage disease type II: results of a phase I/II clinical trial. Genet Med 2001;3:132-138.

3. Nicolino M, Byrne B, Wraith JE, et al. Clinical outcomes after long-term treatment with alglucosidase alfa in infants and children with advanced Pompe disease. Genet Med 2009;11:210-219.

4. Kishnani PS, Corzo D, Nicolino M, et al. Recombinant human acid [alpha] glucosidase: major clinical benefits in infantile-onset Pompe disease. Neurology 2007;68:99-109.

5. Kishnani PS, Goldenberg PC, DeArmey SL, et al. Cross-reactive immunologic material status affects treatment outcomes in Pompe disease infants. Mol Genet Metab 2010;99:26-33.

6. Banugaria SG, Prater SN, Ng YK, et al. The impact of antibodies on clinical outcomes in diseases treated with therapeutic protein: lessons learned from infantile Pompe disease. Genet Med 2011;13:729-736.

7. Kishnani PS, Corzo D, Leslie ND, et al. Early treatment with alglucosidase alpha prolongs long-term survival of infants with Pompe disease. Pediatr Res 2009;66:329-335.

8. Kishnani PS, Nicolino M, Voit T, et al. Chinese hamster ovary cell-derived recombinant human acid alpha-glucosidase in infantile-onset Pompe disease. J Pediatr 2006;149:89-97.

9. Myozyme. [Package insert]. Genzyme Corporation: Cambridge (MA), 2006.

10. Hunley TE, Corzo D, Dudek M, et al. Nephrotic syndrome complicating alpha-glucosidase replacement therapy for Pompe disease. Pediatrics 2004;114:e532-e535.

11. Banugaria SG, Patel TT, Mackey J, et al. Persistence of high sustained antibodies to enzyme replacement therapy despite extensive immunomodulatory therapy in an infant with Pompe disease: need for agents to target antibody-secreting plasma cells. Mol Genet Metab 2012;105:677-680

advance online publication 9 August 2012. doi:10.1038/gim.2012.81 